\title{
Risk of Human-to-Wildlife Transmission of SARS-CoV-2
}

Sophie Gryseels*1,2,3, Luc De Bruyn ${ }^{3,4}$, Ralf Gyselings ${ }^{4}$, Sébastien Calvignac-Spencer ${ }^{5}$, Fabian H. Leendertz ${ }^{5}$, Herwig Leirs ${ }^{3}$

(1) Department of Microbiology, Immunology and Transplantation, Rega Institute, KU Leuven, Belgium

(2) Department of Ecology and Evolutionary Biology, University of Arizona, USA

(3) Department of Biology, University of Antwerp, Belgium

(4) Research Institute for Nature and Forest (INBO), Belgium

(5) Robert Koch Institute, Germany

Keywords: mammals; wildlife; SARS-CoV-2; human-to-wildlife transmission; COVID-19

*Correspondence: sophiegryseels@gmail.com

\begin{abstract}
It has been a long time since the world has experienced a pandemic with such a rapid devastating impact as the current COVID-19 pandemic. The causative agent, the Severe Acute Respiratory Syndrome coronavirus 2 (SARS-CoV-2) is further unusual in that it appears capable of infecting many different mammal species. As a significant proportion of people worldwide are infected with SARS-CoV-2 and may spread the infection unknowingly before symptoms occur or without any symptoms ever occurring, there is a nonnegligible risk of humans spreading SARS-CoV-2 to wildlife, in particular mammals. Because of SARSCoV-2's evolutionary origins in bats and reports of humans transmitting the virus to pets and zoo animals, regulations for prevention of human-to-animal transmission have so far focused mostly on these animal groups. Here, we summarize recent studies and reports that show that a wide range of distantly related mammals are likely susceptible to SARS-CoV-2 and that susceptibility or resistance to the virus is in general not predictable, or only to some extent, by phylogenetic proximity to known susceptible or resistant hosts. In the absence of solid evidence on the SARS-CoV-2 susceptibility/resistance for each of the $\sim 6,500$ known mammal species, we argue that sanitary precautions should be taken when interacting with any mammal species in the wild. Preventing human-to-wildlife SARS-CoV-2 transmission is important for protecting these (sometimes endangered) animals from disease, but also to avoid establishment of novel SARS-CoV-2 reservoirs in wild animals. The risk of repeated re-infection of humans from such a wildlife reservoir could severely hamper SARS-CoV-2 control efforts. For wildlife fieldworkers interacting directly or indirectly with mammals, we recommend sanitary precautions such as physical distancing, wearing face masks and gloves, and frequent decontamination, which are very similar to regulations currently imposed to prevent transmission among humans.
\end{abstract}




\section{Introduction}

Humans across the world are currently facing one of the most impactful pandemics in history. By May $10^{\text {th }}$, about 280,000 deaths and over 4 million confirmed COVID-19 cases have been reported worldwide, of which about 2.5 million are people with currently ongoing infection. These numbers are a large underestimation as many people with mild to moderate symptoms or no symptoms (i.e. non-hospitalized cases) have not been tested [1]. A non-negligible proportion of people can thus be expected to be infected with SARS-CoV-2, the coronavirus that causes COVID-19 at a single moment in time. This virus is very efficiently transmitted via saliva and nasal droplets, which either directly come in contact with mouth and nose epithelia or indirectly through touching contaminated surfaces, and can travel via exhaled air [2]. Infected people are already infectious days before the onset of COVID-19 symptoms, and a large proportion of infected people remain asymptomatic yet infectious for several days [3-5]. While strict social distancing measures have substantially reduced transmission in many areas, complete world-wide eradication is probably unfeasible any time soon. SARS-CoV-2 might continue to circulate in human populations, probably with oscillations in prevalence, for a considerable time [6].

Transmission of human pathogens to non-human animals, including wildlife, occurs more regularly than often thought [7,8]. SARS-CoV-2 appears to have a striking ability to infect a broad range of distantly related mammals. In combination with its high transmissibility and its presence in a significant number of (potentially asymptomatic) people across the world, this possibly creates a dangerous situation of humans unknowingly transmitting the virus to susceptible mammal populations.

Here, we discuss the current evidence of the mammalian evolutionary origins of human coronaviruses, the range of animals SARS-CoV-2 could be able to infect, the potential pathological effects on these animals, and the likelihood and potential consequences of sustained transmission of SARS-CoV-2 among wildlife populations. We further summarize precautions that any person in direct or indirect contact with wild mammals, such as wildlife biologists, conservationists, forestry workers, people working in wildlife rehabilitation centers and people involved in wildlife tourism can take

\section{What are coronaviruses and where do they come from?}

Coronaviruses $(\mathrm{CoV})$ are RNA viruses of the subfamily Coronavirinae, in which four genera can be distinguished: Alpha-, Beta-, Gamma- and Deltacoronavirus. Gamma and delta CoV have been found mostly in avian hosts. Alpha and beta CoV appear largely associated with bats, but also other mammals [9]. Many alpha and beta $\mathrm{CoV}$ lineages appear to have a long evolutionary history mostly confined to particular chiropteran genera, though spillovers (i.e., cross-species transmission events) to other host species, including humans and livestock animals, frequently occur with apparent less long onwards transmission [10].

Seven $\mathrm{CoV}$ are known to infect or have infected humans, all with an ancestry in wildlife mammals. Four of these, the alpha HCoV-NL63 and HCoV-229E and beta HCoV-OC43 and HCoV-HKU1, commonly circulate in people around the world with seasonal oscillations in prevalence and generally cause mild respiratory symptoms such as the common cold [11]. These viruses are assumed to have an evolutionary origin in either bats or rodents [12]. Nevertheless, closer relatives of $\mathrm{HCoV}-229 \mathrm{E}$ are found in alpacas and dromedaries, and the sister clade to $\mathrm{HCoV}-\mathrm{OC} 43$ comprises viruses infecting a wide range of mammals, primarily artiodactyls but also dogs, suggesting these viruses could have passed a significant proportion of their evolutionary time in non-bat and non-rodent hosts before spilling over to humans $[12,13]$. 
The first coronavirus known to have inflicted severe disease in humans was the Severe Acute Respiratory Syndrome coronavirus (SARS-CoV-1), which emerged in humans in 2002-2003, with a total of \pm 8000 confirmed infections and a $\pm 10 \%$ case fatality rate. SARS-CoV-1 likely has an evolutionary origin in horseshoe bats (Rhinolophus spp.) [14], but as this virus was also found to circulate in captive masked palm civets (Paguma larvata) and raccoon dogs (Nyctereutes procyonoides) in markets and some farms, these species may have acted as an intermediate host $[15,16]$. It is also possible, however, that rather in reverse, humans acted as an intermediate host for these carnivores.

Middle-Eastern Respiratory Syndrome virus (MERS-CoV) first emerged in 2012 in humans and has a \pm $35 \%$ case fatality rate. While also having an evolutionary origin in bats (likely Vespertilionidae) [17, 18], it is clear that humans repeatedly acquire MERS through close contact with dromedaries without sustained human-to-human transmission [19]. Dromedaries were likely infected by a MERS ancestor a few decades ago, either directly from bats or via another intermediate host, and can be considered the MERS reservoir now [20].

The novel coronavirus that emerged in humans in December 2019, SARS-CoV-2, is phylogenetically closely related to SARS-CoV-1, without being its closest relative [21]. Rather each virus form subclades with coronaviruses mostly found in horseshoe bats [21]. SARS-CoV-2 is less deadly than SARS-CoV-1 but has a higher transmission rate, further facilitated by asymptomatic and pre-symptomatic transmission [22]. Hence, what started as an epidemic in Wuhan, China, quickly escalated to a pandemic.

As in all coronaviruses, the protein that forms the "spikes" on the virion surface, the spike (S) protein, mediates the recognition and entry into host cells. The receptor binding domain (RBD) of the S protein folds into such a configuration that it can bind to a particular protein on the surface of host cells, in the case of SARS-CoV-2 and other SARS-related CoV the angiotensin-converting-enzyme-2 (ACE2) [23]. The genetic variation of the ACE2 gene among vertebrate species results in variation in chemical properties of the protein. These variations can affect the efficiency with which RBD binds to ACE2, and therefore ACE2 is a major determinant in a species' susceptibility to SARS-CoV-2 [23]. Unlike SARS-CoV-1, SARS-CoV2 further contains a polybasic cleavage site (PCS), an insertion of a few residues that allows host enzymes to cleave the $S$ protein for more efficient cell entry [24].

It is not known which animal species directly infected the first human of the COVID-19 pandemic, as no coronavirus similar enough to SARS-CoV-2 has yet been found in a non-human source [25]. The closest relative to SARS-CoV-2, strain RATG13, was recovered from an intermediate horseshoe bat, Rhinolophus affinis [21]. Its genome is about $96 \%$ identical to SARS-CoV-2, but crucially, the gene sequence coding for the RBD differs substantially from SARS-CoV-2 [21]. Surprisingly, the SARS-CoV-2 RBD is more closely related to a strain of $\mathrm{CoV}$ found in two Malaysian pangolins (Manis javanica). That strain forms a sister lineage to the SARS-CoV-2-RATG13-clade in all other gene regions [26]. However, in some gene regions, two CoV found in $R$. sinicus are closer to the SARS-CoV-2-RATG13 lineage and again in other regions one from $R$. malayanus is closest [27, 28]. The pangolin CoV did not contain a PCS, but such a rare insertion was present in the $\mathrm{CoV}$ from $R$. malayanus [28]. These kind of mosaic genomes and complicated phylogenetic relationships are not unusual for coronaviruses, as they are prone to recombination, where genetic material of different ancestry is exchanged when a host is infected with two distinct CoV strains [11]. A complex history involving several recombination events in natural hosts was also proposed for SARS-CoV-1 [14]. Phylogenetic analyses taking these complex mosaic ancestries into account and using all known SARS-like CoV, of which most are retrieved from horseshoe bats but also includes a second lineage from Malaysian pangolins, suggested that SARS-CoV-2 is itself not the product of recent recombination between known $\mathrm{CoV}$ strains [27]. It likely diverged in its current genomic form from a 
common ancestor with RATG13 40 to 70 years ago [27]. This stretch of evolutionary history could have taken place solely in its natural (horseshoe bat) reservoir or (partly) in another unidentified natural host [27].

\section{A large number of mammal species are at risk of acquiring SARS-CoV-2}

Various pieces of evidence suggest that SARS-CoV-2 is probably able to infect and be transmitted among many mammal species. Box 1 provides an overview of the current state of the still rapidly expanding literature on SARS-CoV-2's potential host range.

Natural observations and infection experiments unequivocally show that SARS-CoV-2 is able to infect and be transmitted among at least cats, ferrets, American mink and hamsters. Tigers, lions and macaques are susceptible but it is unknown, though not unlikely, if transmission would occur within these species. Dogs, Egyptian fruit bats, tree shrews and common marmosets also appear susceptible but unlikely to successfully transmit the virus sustainably onwards. Indirect evidence based on in vitro assays and in silico host-cellreceptor binding modelling further show that probably most Old-World primates are susceptible, as well a high number of distantly related mammal species, ranging from sheep to orcas and with examples from almost every mammalian order. Of note, several species predicted not to be susceptible belong to the orders or families of mammals with known SARS-CoV-2 susceptible species. For example, most New-World primates might not be susceptible, and while hamsters and Peromyscus cricetids might be susceptible, house mice and Norway rats are perhaps not. Based on the above-average genetic variation in the ACE2 gene in bats, inter-species variation in SARS-CoV-2 susceptibility might be particularly high among bats.

SARS-CoV-2 is thus clearly able to infect a wide range of mammal species, some of which are only distantly related to each other, and it is not possible to predict the susceptibility of a species based only on its phylogenetic proximity to humans, horseshoe bats, cats or pangolins. As it will be impossible to individually determine susceptibility for all $~ 6,500$ known mammal species [29], people interacting directly or indirectly with any mammal species should take sanitary precautions to prevent SARS-CoV-2 transmission to wildlife. The U.S. Fish and Wildlife Service and the Bat Specialist Group of the IUCN Species Survival Commission (BSG) have already recommended suspending all fieldwork that involves direct interactions with bats (https://www.iucnbsg.org/). Similarly, the Great Ape Health Consortium urges cancelling all great ape tourism and reducing field research, given these animals' close phylogenetic relatedness with humans and dire conservation status [30]. Several national disease control centers recommend co-quarantining pets with owners showing COVID-19 symptoms, and avoid direct contact to protect their pet from infection. Organizations of zoo veterinarians recommend caretakers to wear protective clothing to avoid infecting zoo animals such as primates and large felines [31]. With the current evidence of SARS-CoV-2 being able to infect and spread among many distantly related mammal species, fieldworkers interacting with any wild mammal species should take appropriate precautions to avoid transmission of SARS-CoV-2 to wildlife.

Field-based studies with wild mammals do not necessarily have to be suspended during the COVID-19 pandemic, as long as relatively straightforward sanitary precautions are taken. Such precautions could largely be the same as those imposed in human populations (https://www.who.int/emergencies/diseases/novel-coronavirus-2019/advice-for-public/): practice physical distancing and decontaminate surfaces that other animals may contact. In situations in which physical distancing is not possible, again the same precautions as in the human world can apply: wear a face mask and decontaminate surfaces in direct or indirect contact with your body. These precautions and protective equipment are not necessarily the same as those used when protecting oneself against a variety of infections 
carried by wild animals. In Box 2, we provide an overview of measures to protect wildlife from human infections and how these may differ from some standard procedures.

We realize that not only wild mammals but also captive, domestic and pet mammals can be at risk for acquiring SARS-CoV-2 infection from humans. However, the set of precautions necessary to prevent transmission in these situations may be different from what we propose to prevent transmission to wildlife, and likely require a multidisciplinary action plan that is beyond the scope of our expertise. Therefore, in this manuscript we solely focus on prevention of human-to-wildlife transmission and discuss only the consequences specific to situations in which wild mammals would acquire SARS-CoV-2 infection.

\section{What could be the consequences of human-to-wildlife transmission of SARS- CoV-2?}

\section{Disease in wildlife}

In humans, COVID-19 symptoms range very widely, including mild respiratory problems, gastro-intestinal issues, headaches, severe acute respiratory syndrome, and no symptoms at all [4, 32]. The infection fatality rate of COVID-19 in humans appears to be around $1 \%$ or less, but varies widely with age and co-morbidities $[33,34]$. This percentage could still change with better knowledge on the proportion of undocumented infections. The few examples of natural and experimental infections in macaques, hamsters, cats, tigers and lions suggest similar symptoms as in mild or moderate human cases, but an increased mortality was noted during SARS-CoV-2 outbreaks in mink farms (see Box 1). If there had been a severe pathogenicity in pets such as cats and dogs we assume this would have been noticed already, implying indeed at most a relatively mild disease in these species. The human coronavirus HCoV-OC43, which causes mild respiratory symptoms in humans, also caused mild respiratory symptoms in a population of habituated chimpanzees when this infection was introduced via humans [35]. Other mild human respiratory viruses transmitted to great apes have however led to severe disease and mortality [36]. An alphacoronavirus that commonly circulates in domestic cats spread efficiently among all 60 cheetahs in a safari park, causing severe disease and a high fatality rate in the cheetahs while infected lions did not show any overt symptoms [37].

Because of unique features in their immune system, it has been hypothesized that bats display no or only mild symptoms from many viral infections [38]. Bats from which SARS-related coronaviruses have previously been detected have not been reported to be ill, though a thorough assessment of an animal's health status is almost never performed in surveillance studies. Furthermore, if the symptoms were to range as much as with COVID-19 in humans, it is possible only the asymptomatic or mildly symptomatic bats are captured and sampled, while individuals with more severe disease are always missed during surveillance.

Not only direct mortality or severe disease is a concern. Wild animals often live on the edge of survival, so even a mild disease may result in lower survival or reproduction probabilities. Natural stressful situations, such as food shortages and co-infections may also pre-dispose them for a more severe disease. We could not find reports on sub-lethal coronavirus disease in wildlife but information from domesticated animals may be useful. Equine coronavirus disease has a low mortality rate among horses but causes clinical problems like leukopenia or metabolic disturbances that would have serious secondary consequences in a wildlife situation [39]. Porcine epidemic diarrhea virus considerably reduces reproductive performance of sows [40]. Even without apparent disease symptoms, virus infections may have important fitness consequences: although Puumala hantavirus does not cause clinical illness in its natural host, the bank vole Myodes glareolus, winter survival of infected animals is affected and there are age-dependent effects on reproduction $[41,42]$ 
As such, it is difficult to predict the pathogenicity of SARS-CoV-2 in new mammalian hosts in the wild. Lacking evidence for absence of morbidity or mortality for most mammalian species, great caution is especially needed to prevent SARS-CoV-2 transmission to endangered species.

\section{Establishment of a novel reservoir}

As several mammal species are susceptible to SARS-CoV-2 and the virus appears to be transmitted easily among humans and experimentally infected susceptible animals (see Box 1), SARS-CoV-2 has the potential to spread very fast in a wild mammal population. Eventually, if the virus can circulate uninterruptedly for some time, a new non-human reservoir can be established. This scenario would pose a significant hurdle for efforts in controlling SARS-CoV-2 in the human population. The new, much less controllable, wildlife source would have the potential to start new epidemics in humans, even when the transmission among humans has been stopped in an area. As SARS-CoV-2 is a measurably evolving RNA virus that is furthermore prone to recombination with other coronaviruses, significant evolutionary change (both neutral and maybe to a lesser extent adaptive) can be expected when circulating for months or years in a new reservoir. While it is not possible to predict which evolutionary trajectory such a new SARS-CoV-2 wildlife lineage would take, one could speculate that this evolution could by chance entail changes in the dominant epitopes targeted by the immune response induced by vaccines currently under development, risking that future vaccinated people would be less protected against the newly evolved wildlife strain. On the other hand, one could also speculate that as the virus adapts to a new host species, it would evolve to a version less capable of infecting humans.

Establishment of a SARS-CoV-2 reservoir in wildlife populations could furthermore lead to negative and even hostile perceptions of these species among humans. Bats already have a bad connotation in different parts in the world [43]. This aversion appears to increase since bats are mentioned as the probable source of the present pandemic in the (social) media. Even in China where bats are traditionally symbols of good luck and happiness, they are more at risk since the COVID-19 pandemic [44]. Furthermore, the known presence of a virus dangerous to humans in particular mammals could severely complicate field research on these species and tourism activities, both often greatly needed for their conservation.

The likelihood of SARS-CoV-2 successfully spreading in a population starting from e.g. a single infected animal depends on how long the animal is infectious and on the population-ecological and behavioral characteristics that determine the contact frequency between animals. For example, the infection is less likely to spread among animals with a solitary lifestyle than among animals that live in herds or large family groups. Contact frequencies can vary spatially and seasonally, e.g. in breeding versus non-breeding season, or due to variations in population density through variation in resources [45-47]. The sustainability of transmission chains in the long term further depends on the overall abundance of susceptible animals. The latter in turn depends on population size(s), the turnover rate in the population due to births of new susceptibles, the proportion of animals that are already immune (and the duration of that immunity) and the probability that any co-occurring related coronaviruses induce cross-immunity, and the connectivity between (meta-)populations [48-51]. With a virus like SARS-CoV-2 that can infect a wide range of mammal species, such connected meta-populations can extend across different species in the community. This would enlarge the actual abundance of susceptible individuals in comparison to a single species, thus increasing the probability of long-term persistence of SARS-CoV-2 transmission. Furthermore, through such inter-species connections in (indirect) contact networks, the infection could (repeatedly) reach individuals of species of which population densities and contact frequencies would be too low to maintain SARS-CoV-2 transmission. As such, a cascade of transmission chains among interconnected populations of different SARS-CoV-2-susceptible species could ultimately also reach endangered species. A similar 
phenomenon is documented for Yersinia pestis plague in prairie dog colonies in the United States, which repeatedly reaches and kills the critically endangered black-footed ferret [52]. In fact, plague is an excellent example of how a pathogen can act as an invasive transformer species affecting the stability of the ecosystem [53].

With the many variables involved, it is difficult to quantify the risk that one individual animal infected by a human continues to spread the infection among populations and establish a new SARS-CoV-2 reservoir. From experience of infections emerging in human populations, we can qualitatively assess that this risk is actually rather low and prone to stochasticity. Humans have frequent interactions with wildlife through hunting, the wildlife-meat trade, animal (wet) markets and occupational activities (e.g. bat guano harvesters, forestry workers, and wildlife researchers), and have a unique global contact network containing wellconnected high-density clusters. Still, the viral infections that humans probably quite regularly acquire from wildlife only very rarely lead to long human-to-human transmission chains [54]. Most wild mammal populations or even multi-species communities are perhaps less suited for sustained SARS-CoV-2 transmission than human populations. On the other hand, the number of contacts from the same human reservoir to wild mammal species may be higher than the reverse situation, in turn leading to an increased probability that at least one of these contacts leads to successful onwards transmission. In any case, even if the risk is low, the stakes of sustained SARS-CoV-2 transmission in wildlife are very high. The relatively simple precautions outlined in Box 2 can substantially reduce the risk that wildlife fieldworkers transmit the infection to wild mammals.

\section{Conclusion}

The likelihood that mammologists, conservationists, wildlife field researchers, or anybody interacting directing or indirectly with wild mammals, initiate a chain of SARS-CoV-2 transmission among wild mammal populations is not negligible and probably higher than with other common human viruses. Indeed, SARS-CoV-2 presents unique features: it is currently present in a significant proportion of humans worldwide; it is highly transmissible through direct and indirect contact and via airborne droplets; many people are infectious without symptoms (therefore quarantining only when sick is not sufficient to prevent transmission); finally, the virus is able to infect a wide range of distantly related mammals and predicting which animal species are susceptible seems challenging. Combined with the potential devastating impact on both humans and wild mammalian populations that sustained SARS-CoV-2 transmission in wildlife would have, we urge taking sensible sanitary precautions when in contact with any mammal species to reduce this risk of human-to-wildlife SARS-CoV-2 transmission as much as possible.

\section{Acknowledgements}

We thank Bruno Gryseels for useful feedback on the manuscript. SG is supported by an OUTGOING [PEGASUS] $^{2}$ Marie Skłodowska-Curie Fellowship (12T1117N) of the Research Foundation - Flanders (FWO). HL is part the University of Antwerp Center of Excellence VAX-IDEA. 


\section{Box 1. Which animals are (not) susceptible to SARS-CoV-2?}

Here we provide an overview of the current, and rapidly expanding, pieces of evidence that show that many different distantly related mammals are susceptible to SARS-CoV-2 and are able to transmit the virus onwards, while others appear resistant. We can classify current evidence of SARS-CoV-2 susceptibility into four categories: infections that occurred naturally, animal infection experiments, in vitro infection experiments with cell lines and predicted molecular recognition of SARS-CoV-2 spike protein with host species ACE2 proteins. Note that many of the studies mentioned below have not yet been peer-reviewed and have only been described in press released or in preprint manuscripts on preprint websites, and should thus be interpreted with caution.

\section{Natural infections}

In mid-March, a veterinary diagnostics company, tested for presence of SARS-CoV-2 in 4000 pets across USA. None were positive, but this testing occurred at a time when there were only about 3000 confirmed human cases in USA [55]. Two asymptomatic pet dogs in Hong Kong were found SARS-CoV-2 positive during a small investigation where $17 \mathrm{dogs}$ and 8 cats were tested from households with confirmed COVID19 cases or close contacts of such cases [56]. Five pet cats (one in Belgium, one in Hong Kong, two in New York, one in France) symptoms were confirmed with ongoing SARS-CoV-2 infection [56-62]. They displayed mild respiratory symptoms and some also had digestive problems. For four of these cats their owners were COVID-19 positive, but in one free-roaming cat in New York the owner was negative but a neighbor was positive. On the other hand, in a student community where 13 out of 20 people likely had COVID-19, none of the 9 cats and 12 dogs living with them were virus-positive or displayed anti-SARS$\mathrm{CoV}$ antibodies [63]. In the city of Wuhan, China, where the epidemic likely started, 102 pet cats were tested for the presence of neutralizing anti-SARS-CoV-2 antibodies of which $15 \%$ were positive [64]. These serological tests indicate a past infection from which the cats recovered. Thirty-nine cats sampled before the start of the outbreak (i.e. before December 2019) were all negative.

Five co-housed tigers and three lions in the Bronx Zoo (USA) acquired SARS-CoV-2 infection from a COVID-19 positive caretaker [65]. The initial transmission likely occurred via indirect contact (i.e. via contaminated surfaces) with the caretaker but potentially subsequent transmission occurred among the animals within the same enclosure. All but one tiger, who remained asymptomatic despite testing positive for SARS-CoV-2 in its faeces, displayed a dry cough and wheezing [65].

SARS-CoV-2 spread among American mink (Neovison vison) individuals in four different fur farms in the Netherlands, with the initial infections arising from caretakers with COVID-19-like symptoms [66]. The animals showed gastro-intestinal and respiratory symptoms and an increased mortality rate was noted.

\section{Animal infection experiments}

SARS-CoV-2 did not replicate in intranasally inoculated outbred laboratory mice (ICR) unless they were genetically modified to express human ACE2 [67]. This could suggest wild house mice (Mus musculus domesticus) are not susceptible the virus, but the susceptibility of inbred lab mouse strains BALB/c, C57BL6 and 129S to SARS-CoV-1 suggests murine susceptibility to SARS-CoV-2 requires further investigation. SARS-CoV-2 also did not replicate in domestic ducks, chickens and pigs [68, 69]. SARSCoV-2 did replicate in inoculated domestic dogs, ferrets and domestic cats. In contrast to cats and ferrets, replication and viral shedding were low in dogs, and no transmission to co-housed naïve dogs occurred [68]. The infected cats transmitted the virus to $2 / 6$ non-inoculated cats, in one instance with evidence for airborne transmission [68]. Infection of ferrets and efficient ferret-to-ferret transmission, including some 
airborne, was demonstrated in several studies [68-71]. Rhesus and crab-eating macaques (Macaca mulatta and M. fascicularis) and Syrian hamsters (Mesocricetus auratus), often used model species in biomedical research, are also permissive to infection. The macaques develop similar viral shedding dynamics and symptoms as in human COVID-19 patients, with more severe symptoms in M. mulatta than M. fascicularis [72-75]. The Syrian hamsters transmitted the virus to co-housed hamsters. SARS-CoV-2 was able to replicate in some but not all intranasally inoculated tree shrews (Tupaia belangeris), another emerging lab model species. Fever was the only clinical sign some individuals displayed [76]. SARS-CoV-2 genetic material was detectable in blood and excreta of intranasally inoculated common marmosets (Callithrix jacchus) but not in organs, and they remained visibly asymptomatic [75]. Lastly, a press release reports nasally inoculated Egyptian fruit bats (Rousettus aegypticus) "became infected, but did not show any symptoms of disease and did not infect their fellow animals efficiently" [69].

\section{In vitro cell culture infection experiments}

The ability of a virus to infect cultured cells in vitro provides evidence for its ability to infect tissues of the animal species from which these cells are derived. This does not necessarily mean the virus would be able to replicate efficiently enough in the actual bodies of these animal species or transmission to other individuals would be possible. For most infection experiments with laboratory cell lines mentioned below, an engineered chimera of a standard laboratory virus strain in which (part of) SARS-CoV-2 spike protein is inserted is used, as this method has been shown to model infection with the real virus into cells quite well $[77,78]$. Many cell-culture lines, however, do not (sufficiently) express the ACE2 proteins that SARS-like viruses need to bind to initiate infection. This for example explains why SARS-CoV-1 and SARS-CoV-2 failed to infect the RhiLu/1.1 cell line derived from the Halcyon horseshoe bat $R$. alcyone [77]. Therefore, in vitro studies rely on modifying commonly used cell lines to express ACE2 proteins (derived from several species), as such only reflecting the ability of SARS-CoV-2 to enter cells with ACE2 protein of a particular species but not necessarily whether intracellular replication would occur in that species. These in vitro studies must thus be interpreted with care.

We found two studies in which HeLa cells were modified to express the ACE2 protein of a total of 51 different mammal species, and in 45 of them SARS-CoV-2 was able to infect these cells [24, 79]. This proportion is probably biased, as in the (not yet peer-reviewed) study in which 49 of the 51 species were investigated, purposely species were selected of which the ACE2 sequence was already estimated to be able to bind to SARS-CoV-2's RBD based on structure homology models [79]. Nevertheless, as this large number of potentially susceptible mammals are scattered across the mammalian phylogeny, including e.g. Chinese horseshoe bats ( $R$. sinicus), white-footed mice (Peromyscus leucopus), beluga whales (Delphinapterus leucas), giant pandas (Ailuropoda melanoleuca) and white rhinoceros (Ceratotherium simum), these in vitro studies further support that SARS-CoV-2 is able to infect a wide range of distantly related mammals. SARS-CoV-2 was able to infect cells transfected with ACE2 of domestic pigs but apparently not in experimentally inoculated live pigs [68] (see above), showing a discrepancy between inference from in vitro and in vivo studies.

\section{In silico 3D structure modelling of ACE2-spike interactions}

The structure and 3D configuration of ACE2 homologs of different vertebrate species can be modeled based on comparison with the known human ACE2 structure. Theoretically, one can then infer whether or not the RBD of SARS-CoV-2 would be able to bind to that species ACE2 protein, if its sequence is available. The extent at which we can subsequently infer whether a species is SARS-CoV-2 susceptible, let alone the possibility of transmission between actual bodies of the species, is still largely a black box. The uncertainty increases with increasing divergence from the human ACE2 sequence (of which the 3D structure in 
complex with SARS-CoV-2 has actually been determined [80-82]) [83]. The binding capacity of ACE2 with SARS-CoV-2 does not necessarily decrease with more divergence from humans. In the case of SARS$\mathrm{CoV}-1$, it was shown that single residue changes in ACE2 can alter the binding efficiency with SARS-CoV1's RBD significantly [84]. The many manuscripts currently appearing in which such species susceptibility predictions are solely based on ACE2 sequence analyses should thus be interpreted with caution.

Still, the main conclusion from in silico ACE2-RBD binding studies is in line with evidence from natural and experimental infections: a wide range of mammals may be susceptible and only to some extent is phylogenetic proximity to a known susceptible host a prediction of a species susceptibility. All investigated catarrhine primates (apes and Old World monkeys) have identical residues at the sites thought to interact with SARS-CoV-2's RBD, making it likely that all these species are susceptible to SARS-CoV-2 [83, 85, 86]. The ACE2 in New World monkeys contains some key differences that might lead to resistance according to structure models $[83,85,86]$. However, as mentioned above, SARS-CoV-2 replication occurred in at least one species of New World monkey after experimental infection (in a not-yet peer reviewed study), though with rapid clearance and no disease symptoms [75]. Structure models further also indicate that mammals with inferred binding compatibility with SARS-CoV-2's RBD are scattered across the mammalian tree $[79,83,85-88]$. Unpredictability from phylogenetic proximity could be especially the case among Chiropteran species, as the available data so far shows that the diversity of ACE2 residues implicated in contacting SARS-CoV's RBDs is substantially greater among bat species than among species in other mammalian orders $[83,86,89]$. Therefore, despite the likely evolutionary ancestry of SARS-like viruses in horseshoe bats, the disproportionally large chiropteran ACE2 diversity suggests that the variation in SARS-CoV-2 susceptibility may perhaps vary much more among bat families/genera/species than among clades of other mammalian orders.

\section{Box 2: Guidelines to prevent SARS-CoV-2 transmission to wildlife}

Many fieldworkers interacting with live or dead wild animals are accustomed to taking protective measures to protect oneself from animal infections (e.g. when handling rodents in hantavirus endemic areas) or to prevent cross-contaminating infections between individual animals or populations (e.g. when working with bats in areas affected by white-nose syndrome). It requires a somewhat different set of precautions when avoiding transmitting one's own viral infections to animals. Many other fieldworkers have little or no experience with biosafety precautions in field settings. The sanitary measures described below require some practice in mock situations before their implementation in the field to become accustomed to appropriate face mask wearing and the routine of regular decontamination of any material that could come in contact with an animal.

Fieldwork with any mammal should be suspended when the fieldworker is coughing, sneezing or generally feeling ill. As SARS-CoV-2 can be transmissible before the onset of symptoms or in the absence of symptoms, one should be aware that one can be infected with and transmit SARS-CoV-2 without knowing it.

Similar to precautions taken among people to avoid spreading SARS-CoV-2, one should keep a physical distance with the study animal. WHO recommends at least one meter distance between people, but other countries impose longer distances in their regulations. The IUCN primate specialist group recommends at least $7 \mathrm{~m}$ distance between humans and great apes [90]. The appropriate distance depends on the behavioral characteristics of the species under observation; could it be expected to quickly move closer to the person? 
Physical distancing is feasible for many wildlife observational studies, such as monitoring populations through counting individuals from a distance. Note that even in these situations, if one needs to come closer to surfaces that might later be in contact with the animal, one must wear a face mask appropriately to avoid leaving saliva or nasal droplets on those surfaces. Similarly, if one needs to touch surfaces that might later be in contact with the animal, wear clean latex or nitrile gloves and decontaminate these surfaces. In particular, face masks and clean gloves must be worn when setting up bait stations for e.g. camera traps and observational studies, to avoid contamination of the material that mammals will be in contact with.

If essential fieldwork requires direct contact or coming closer to live mammals than considered safe, one should follow strict measures:

- Wear clothes or a coverall that have been freshly washed. Change to such clean clothes before every field session.

- Wash hands and face before every field session.

- Decontaminate all material and surfaces that may later be in contact with the animal or that you leave behind in the field and that an animal may touch. Use an appropriate disinfectant such as $70 \%$ ethanol, bleach solution ( $40 \mathrm{~mL}$ household bleach in $1 \mathrm{~L}$ of water), or a peroxygen compound like Virkon.

- Wear an appropriate face mask covering mouth and nose (see below).

- Wear clean latex or nitrile gloves.

\section{Which face masks to wear and how to handle them properly?}

It is important to keep in mind that the aim here is to prevent transmission of one's own pathogens to animals rather than the reverse. To avoid spreading our own saliva or nasal droplets, a properly made cloth mask or a disposable surgical mask are appropriate, much like surgeons wear to prevent transmitting their respiratory microbes to their patients.

Respirator masks such as FFP2/N95 or FFP3/N99 are more closely sealed and filter finer particles, but are rather meant for protecting oneself from (aerosolized) microorganisms. If such respirator masks must be worn as Personal Protective Equipment (PPE) under particular field work conditions, make sure they do not contain an exhalation valve; such a valve may increase breathing comfort but will allow the outward passage of droplets.

Similarly, most power-aired purifying respirators (PAPRs), often worn as PPE when handling animals potentially infected with very dangerous airborne pathogens, do not protect against spreading an infection. Indeed, in most PAPRs only the incoming air is filtered while the outgoing air, containing human respiratory droplets, is not filtered and even expelled under positive pressure. Therefore, if PAPRs are required for personal protection during field work with mammals that are released alive, make sure the PAPR-hood also contains a filter for the outgoing air.

When using a re-usable cloth mask, make sure it has been decontaminated (e.g. washed at $90^{\circ} \mathrm{C}$ ) before every field session. After taking off and putting a face mask back on during a field session (e.g. when taking a break), the inside of the mask should not be handled, as this contains one's respiratory droplets, and decontaminate your hands afterwards. If several masks are available per day, wear a new clean one every time you change masks.

\section{How to wear gloves properly?}

When putting on latex or nitrile gloves, only the sleeve's edge of the glove should be touched with your bare hands, not touching the rest of the glove. Do not touch your face or items possibly contaminated with 
your respiratory droplets once you are wearing gloves. If this should happen, change gloves or decontaminate them with a disinfectant.

\section{References}

1. Li, R., S. Pei, B. Chen, Y. Song, T. Zhang, W. Yang, and J. Shaman. (2020) Substantial undocumented infection facilitates the rapid dissemination of novel coronavirus (SARS-CoV2). Science.

2. Meselson, M. (2020) Droplets and Aerosols in the Transmission of SARS-CoV-2. New England Journal of Medicine.

3. Gudbjartsson, D.F., et al. (2020) Spread of SARS-CoV-2 in the Icelandic Population. New England Journal of Medicine.

4. Pan, X., D. Chen, Y. Xia, X. Wu, T. Li, X. Ou, L. Zhou, and J. Liu. (2020) Asymptomatic cases in a family cluster with SARS-CoV-2 infection. The Lancet Infectious Diseases. 20(4): 410-411.

5. Sutton, D., K. Fuchs, M. D'Alton, and D. Goffman. (2020) Universal screening for SARS-CoV-2 in women admitted for delivery. New England Journal of Medicine.

6. Kissler, S.M., C. Tedijanto, E. Goldstein, Y.H. Grad, and M. Lipsitch. (2020) Projecting the transmission dynamics of SARS-CoV-2 through the postpandemic period. Science. eabb5793.

7. Messenger, A.M., A.N. Barnes, and G.C. Gray. (2014) Reverse zoonotic disease transmission (zooanthroponosis): a systematic review of seldom-documented human biological threats to animals. PLoS One. 9(2): e89055-e89055.

8. Epstein, J.H. and J.T. Price. (2009) The significant but understudied impact of pathogen transmission from humans to animals. Mount Sinai Journal of Medicine: A Journal of Translational and Personalized Medicine. 76(5): 448-455.

9. Anthony, S.J., et al. (2017) Global patterns in coronavirus diversity. Virus Evolution. 3(1): vex012.

10. Leopardi, S., E.C. Holmes, M. Gastaldelli, L. Tassoni, P. Priori, D. Scaravelli, G. Zamperin, and P. De Benedictis. (2018) Interplay between co-divergence and cross-species transmission in the evolutionary history of bat coronaviruses. Infection, Genetics and Evolution. 58: 279-289.

11. Su, S., G. Wong, W. Shi, J. Liu, A.C. Lai, J. Zhou, W. Liu, Y. Bi, and G.F. Gao. (2016) Epidemiology, genetic recombination, and pathogenesis of coronaviruses. Trends in Microbiology. 24(6): 490-502.

12. Corman, V.M., D. Muth, D. Niemeyer, and C. Drosten. (2018) Hosts and sources of endemic human coronaviruses, in: Advances in virus research. M. Kielian, T.C. Mettenleiter, and M.J. Roossinck, Editors., Elsevier. 163-188.

13. Cui, J., F. Li, and Z.L. Shi. (2019) Origin and evolution of pathogenic coronaviruses. Nature Reviews Microbiology. 17(3): 181-192.

14. Hu, B., et al. (2017) Discovery of a rich gene pool of bat SARS-related coronaviruses provides new insights into the origin of SARS coronavirus. PLoS Pathogens. 13(11): e1006698.

15. Guan, Y., et al. (2003) Isolation and Characterization of Viruses Related to the SARS Coronavirus from Animals in Southern China. Science. 302(5643): 276-278.

16. Kan, B., et al. (2005) Molecular evolution analysis and geographic investigation of severe acute respiratory syndrome coronavirus-like virus in palm civets at an animal market and on farms. Journal of Virology. 79(18): 11892-900.

17. Ithete, N.L., S. Stoffberg, V.M. Corman, V.M. Cottontail, L.R. Richards, M.C. Schoeman, C. Drosten, J.F. Drexler, and W. Preiser. (2013) Close relative of human Middle East respiratory syndrome coronavirus in bat, South Africa. Emerging Infectious Diseases. 19(10): 1697.

18. Anthony, S.J., et al. (2017) Further Evidence for Bats as the Evolutionary Source of Middle East Respiratory Syndrome Coronavirus. Mbio. 8(2). 
19. Dudas, G., L.M. Carvalho, A. Rambaut, and T. Bedford. (2018) MERS-CoV spillover at the camelhuman interface. Elife. 7: e31257.

20. Corman, V.M., N.L. Ithete, L.R. Richards, M.C. Schoeman, W. Preiser, C. Drosten, and J.F. Drexler. (2014) Rooting the phylogenetic tree of middle East respiratory syndrome coronavirus by characterization of a conspecific virus from an African bat. Journal of Virology. 88(19): 1129711303.

21. Zhou, P., et al. (2020) A pneumonia outbreak associated with a new coronavirus of probable bat origin. Nature. 579(7798): 270-273.

22. He, X., et al. (2020) Temporal dynamics in viral shedding and transmissibility of COVID-19. Nature medicine.

23. Wan, Y., J. Shang, R. Graham, R.S. Baric, and F. Li. (2020) Receptor Recognition by the Novel Coronavirus from Wuhan: an Analysis Based on Decade-Long Structural Studies of SARS Coronavirus. Journal of Virology. 94(7).

24. Hoffmann, M., et al. (2020) SARS-CoV-2 cell entry depends on ACE2 and TMPRSS2 and is blocked by a clinically proven protease inhibitor. Cell.

25. Zhang, Y.Z. and E.C. Holmes. (2020) A Genomic Perspective on the Origin and Emergence of SARS-CoV-2. Cell. 181(2): 223-227.

26. Lam, T.T., et al. (2020) Identifying SARS-CoV-2 related coronaviruses in Malayan pangolins. Nature.

27. Boni, M.F., P. Lemey, X. Jiang, T.T.-Y. Lam, B. Perry, T. Castoe, A. Rambaut, and D.L. Robertson. (2020) Evolutionary origins of the SARS-CoV-2 sarbecovirus lineage responsible for the COVID19 pandemic. bioRxiv. 2020.03.30.015008.

28. Zhou, H., et al. (2020) A novel bat coronavirus reveals natural insertions at the S1/S2 cleavage site of the Spike protein and a possible recombinant origin of HCoV-19. bioRxiv. 2020.03.02.974139.

29. Burgin, C.J., J.P. Colella, P.L. Kahn, and N.S. Upham. (2018) How many species of mammals are there? Journal of Mammalogy. 99(1): 1-14.

30. Gillespie, T.R. and F.H. Leendertz. (2020) COVID-19: protect great apes during human pandemics. Nature. 579(7800): 497.

31. EAZWV. (28/04/2020) Science-based facts \& knowledge about wild animals, zoos and sars-cov-2 virus. European Association of Zoo and Wildlife Veterinarians. https://cdn.ymaws.com/www.eazwv.org/resource/resmgr/files/transmissible diseases handbook/ 5th ed transmissible diseases handbook/chapters/covid19 faq v6.1 28 april 20.pdf

32. Guan, W., et al. (2020) Clinical Characteristics of Coronavirus Disease 2019 in China. New England Journal of Medicine. 382(18): 1708-1720.

33. Streeck, H., et al. (2020) Infection fatality rate of SARS-CoV-2 infection in a German community with a super-spreading event. medRxiv. 2020.05.04.20090076.

34. Verity, R., et al. (2020) Estimates of the severity of coronavirus disease 2019: a model-based analysis. The Lancet Infectious Diseases.

35. Patrono, L.V., L. Samuni, V.M. Corman, L. Nourifar, C. Röthemeier, R.M. Wittig, C. Drosten, S. Calvignac-Spencer, and F.H. Leendertz. (2018) Human coronavirus OC43 outbreak in wild chimpanzees, Côte d' Ivoire, 2016. Emerging Microbes \& Infections. 7(1): 1-4.

36. Köndgen, S., et al. (2008) Pandemic Human Viruses Cause Decline of Endangered Great Apes. Current Biology. 18(4): 260-264.

37. Wilkerson, A.J.P., E.C. Teeling, J.L. Troyer, G.K. Bar-Gal, M. Roelke, L. Marker, J. PeconSlattery, and S.J. O'Brien. (2004) Coronavirus outbreak in cheetahs: lessons for SARS. Current Biology. 14(6): R227-R228.

38. Schountz, T., M.L. Baker, J. Butler, and V. Munster. (2017) Immunological Control of Viral Infections in Bats and the Emergence of Viruses Highly Pathogenic to Humans. Frontiers in Immunology. 8(1098). 
39. Berryhill, E., K. Magdesia, M. Aleman, and N. Pusterla. (2019) Clinical presentation, diagnostic findings, and outcome of adult horses with equine coronavirus infection at a veterinary teaching hospital: 33 cases (2012-2018). The Veterinary Journal. 248: 95-100.

40. Furutani, A., T. Kawabata, M. Sueyoshi, and Y. Sasaki. (2018) Assessment of reproductive performance in F1 sows exposed to the porcine epidemic diarrhea virus at different periods of production stage on farms with different hygienic environments. Animal reproduction science. 192: 233-241.

41. Kallio, E.R., H. Helle, E. Koskela, T. Mappes, and O. Vapalahti. (2015) Age-related effects of chronic hantavirus infection on female host fecundity. Journal of Animal Ecology. 84(5): 12641272 .

42. Kallio, E.R., L. Voutilainen, O. Vapalahti, A. Vaheri, H. Henttonen, E. Koskela, and T. Mappes. (2007) Endemic hantavirus infection impairs the winter survival of its rodent host. Ecology. 88(8): 1911-1916.

43. Kingston, T. (2016) Cute, Creepy, or Crispy-How Values, Attitudes, and Norms Shape Human Behavior Toward Bats, in: Bats in the Anthropocene: Conservation of Bats in a Changing World. C.C. Voigt and T. Kingston, Editors., Springer International Publishing: Cham. 571-595.

44. Zhao, H. (2020) COVID-19 drives new threat to bats in China. Science. 367(6485): 1436-1436.

45. Borremans, B., J. Reijniers, N.K. Hughes, S.S. Godfrey, S. Gryseels, R.H. Makundi, and H. Leirs. (2017) Nonlinear scaling of foraging contacts with rodent population density. Oikos. 126(6): 792800.

46. Davis, S., B. Abbasi, S. Shah, S. Telfer, and M. Begon. (2015) Spatial analyses of wildlife contact networks. Journal of the Royal Society Interface. 12(102): 20141004.

47. White, L.A., J.D. Forester, and M.E. Craft. (2017) Using contact networks to explore mechanisms of parasite transmission in wildlife. Biological Reviews. 92(1): 389-409.

48. Reijniers, J., S. Davis, M. Begon, J.A. Heesterbeek, V.S. Ageyev, and H. Leirs. (2012) A curve of thresholds governs plague epizootics in Central Asia. Ecology Letters. 15(6): 554-560.

49. Anderson, R.M., B. Anderson, and R.M. May. (1992) Infectious diseases of humans: dynamics and control. Oxford university press.

50. Keeling, M.J. and B.T. Grenfell. (1997) Disease extinction and community size: modeling the persistence of measles. Science. 275(5296): 65-7.

51. Keeling, M.J. and B.T. Grenfell. (2000) Individual-based perspectives on R(0). J Theor Biol. 203(1): 51-61.

52. Matchett, M.R., D.E. Biggins, V. Carlson, B. Powell, and T. Rocke. (2010) Enzootic plague reduces black-footed ferret (Mustela nigripes) survival in Montana. Vector-Borne and Zoonotic Diseases. 10(1): 27-35.

53. Eads, D.A. and D.E. Biggins. (2015) Plague bacterium as a transformer species in prairie dogs and the grasslands of western North America. Conservation Biology. 29(4): 1086-1093.

54. Woolhouse, M.E., L. Brierley, C. McCaffery, and S. Lycett. (2016) Assessing the epidemic potential of RNA and DNA viruses. Emerging Infectious Diseases. 22(12): 2037.

55. IDEXX. (13/03/2020) Leading Veterinary Diagnostic Company Sees No COVID-19 Cases in Pets Idexx Laboratories. https://www.idexx.com/en/about-idexx/news/no-covid-19-cases-pets/

56. AFCD. (26/03/2020) Pet dog further tests positive for antibodies for COVID-19 virus. Agriculture Fisheries and Conservation Department of the Hong Kong Special Administrative Region. https://www.info.gov.hk/gia/general/202003/26/P2020032600756.htm

57. Sit, T.H.C., et al. (2020) Canine SARS-CoV-2 infection. Preprint from Research Square. rs.3.rs$18713 / \mathrm{v} 1$.

58. FASFC. (22/03/2020) Zoönotisch risico van het SARS-CoV2 virus (Covid-19) bij gezelschapsdieren: infectie van dier naar mens en van mens naar dier. Federal Agency for the Safety of the Food Chain (Belgium). http://www.afsca.be/wetenschappelijkcomite/adviezen/2020/_documents/Spoedraadgeving042020_SciCom2020-07_Covid-19gezelschapdieren_27-03-20.pdf 
59. AFCD. (31/03/2020) Pet cat tests positive for COVID-19. Agriculture Fisheries and Conservation Department of the Hong Kong Special Administrative Region. https://www.news.gov.hk/eng/2020/03/20200331/20200331_220128_110.html

60. USDA. (22/04/2020) Confirmation of COVID-19 in Two Pet Cats in New York. Animal and Plant Health Inspecition Service of the United States Deparment of Agriculture. https://www.aphis.usda.gov/aphis/newsroom/news/sa by date/sa-2020/sars-cov-2-animals

61. WOAH. (22/04/2020) Follow-up report No. 2. World Organization for Animal Health. https://www.oie.int/wahis_2/public/wahid.php/Reviewreport/Review?reportid=34086

62. Zientara, S. (2020-05-01) Coronavirus disease 2019 update (149): France (Île-de-France) animal, cat, owned. ProMED. https://promedmail.org/promed-post/?id=7289409

63. Temmam, S., et al. (2020) Absence of SARS-CoV-2 infection in cats and dogs in close contact with a cluster of COVID-19 patients in a veterinary campus. bioRxiv. 2020.04.07.029090.

64. Zhang, Q., et al. (2020) SARS-CoV-2 neutralizing serum antibodies in cats: a serological investigation. bioRxiv. 2020.04.01.021196.

65. WCS. (22/04/2020) Update: Bronx Zoo Tigers and Lions Recovering from COVID-19. Wildlife Conservation Society. https://newsroom.wcs.org/NewsReleases/articleType/ArticleView/articleId/14084/Update-Bronx-Zoo-Tigers-and-LionsRecovering-from-COVID-19.aspx

66. MANFQ. (08/05/2020) Eerste resultaten onderzoek naar COVID-19 op nertsenbedrijven binnen. Ministery of Agriculture Nature and Food Quality of The Netherlands. https://www.rijksoverheid.nl/ministeries/ministerie-van-landbouw-natuur-envoedselkwaliteit/nieuws/2020/05/08/eerste-resultaten-onderzoek-naar-covid-19-opnertsenbedrijven-binnen

67. Bao, L., et al. (2020) The Pathogenicity of SARS-CoV-1 2 in hACE2 Transgenic Mice. bioRxiv. 2020.02.07.939389.

68. Shi, J., et al. (2020) Susceptibility of ferrets, cats, dogs, and other domesticated animals to SARScoronavirus 2. Science. eabb7015.

69. Friedrich-Loeffler-Institut. Novel Coronavirus SARS-CoV-2: Fruit bats and ferrets are susceptible, pigs and chickens are not. Friedrich-Loeffler-Institut of the Federal Research Institute for Animal Health, Germany. https://www.fli.de/en/press/press-releases/press-singleview/novel-coronavirussars-cov-2-fruit-bats-and-ferrets-are-susceptible-pigs-and-chickens-are-not/

70. Kim, Y.I., et al. (2020) Infection and Rapid Transmission of SARS-CoV-2 in Ferrets. Cell Host \& Microbe.

71. Richard, M., et al. (2020) SARS-CoV-2 is transmitted via contact and via the air between ferrets. bioRxiv. 2020.04.16.044503.

72. Bao, L., et al. (2020) Reinfection could not occur in SARS-CoV-2 infected rhesus macaques. bioRxiv. 2020.03.13.990226.

73. Munster, V.J., et al. (2020) Respiratory disease and virus shedding in rhesus macaques inoculated with SARS-CoV-2. bioRxiv. 2020.03.21.001628.

74. Rockx, B., et al. (2020) Comparative pathogenesis of COVID-19, MERS and SARS in a nonhuman primate model. bioRxiv. 2020.03.17.995639.

75. Lu, S., et al. (2020) Comparison of SARS-CoV-2 infections among 3 species of non-human primates. bioRxiv. 2020.04.08.031807.

76. Zhao, Y., et al. (2020) Susceptibility of tree shrew to SARS-CoV-2 infection. bioRxiv. 2020.04.30.029736.

77. Letko, M., A. Marzi, and V. Munster. (2020) Functional assessment of cell entry and receptor usage for SARS-CoV-2 and other lineage B betacoronaviruses. Nature microbiology. 5(4): 562-569.

78. Becker, M.M., et al. (2008) Synthetic recombinant bat SARS-like coronavirus is infectious in cultured cells and in mice. Proceedings of the National Academy of Sciences. 105(50): 1994419949. 
79. Liu, Y., et al. (2020) Functional and Genetic Analysis of Viral Receptor ACE2 Orthologs Reveals Broad Potential Host Range of SARS-CoV-2. bioRxiv. 2020.04.22.046565.

80. Yan, R., Y. Zhang, Y. Li, L. Xia, Y. Guo, and Q. Zhou. (2020) Structural basis for the recognition of SARS-CoV-2 by full-length human ACE2. Science. 367(6485): 1444-1448.

81. Lan, J., et al. (2020) Structure of the SARS-CoV-2 spike receptor-binding domain bound to the ACE2 receptor. Nature.

82. Shang, J., G. Ye, K. Shi, Y. Wan, C. Luo, H. Aihara, Q. Geng, A. Auerbach, and F. Li. (2020) Structural basis of receptor recognition by SARS-CoV-2. Nature.

83. Frank, H.K., D. Enard, and S.D. Boyd. (2020) Exceptional diversity and selection pressure on 1 SARS-CoV and SARS-CoV-2 host receptor in bats compared to other mammals. bioRxiv. 2020.04.20.051656.

84. Li, W., et al. (2005) Receptor and viral determinants of SARS-coronavirus adaptation to human ACE2. The EMBO Journal. 24(8): 1634-1643.

85. Damas, J., et al. (2020) Broad Host Range of SARS-CoV-2 Predicted by Comparative and Structural Analysis of ACE2 in Vertebrates. bioRxiv. 2020.04.16.045302.

86. Melin, A.D., M.C. Janiak, F. Marrone, P.S. Arora, and J.P. Higham. (2020) Comparative ACE2 variation and primate COVID-19 risk. bioRxiv. 2020.04.09.034967.

87. Luan, J., Y. Lu, X. Jin, and L. Zhang. (2020) Spike protein recognition of mammalian ACE2 predicts the host range and an optimized ACE2 for SARS-CoV-2 infection. Biochem Biophys Res Commun. 526(1): 165-169.

88. Liu, Z., et al. (2020) Composition and divergence of coronavirus spike proteins and host ACE2 receptors predict potential intermediate hosts of SARS-CoV-2. Journal of Medical Virology.

89. Hou, Y., C. Peng, M. Yu, Y. Li, Z. Han, F. Li, L.F. Wang, and Z. Shi. (2010) Angiotensinconverting enzyme 2 (ACE2) proteins of different bat species confer variable susceptibility to SARS-CoV entry. Arch Virol. 155(10): 1563-9.

90. Gilardi, K.V., T.R. Gillespie, F.H. Leendertz, E.J. Macfie, D.A. Travis, C.A. Whittier, and E.A. Williamson. (2015) Best Practice Guidelines for Health Monitoring and Disease Control in Great Ape Populations. Gland, Switzerland: IUCN SSC Primate Specialist Group. 